\title{
A NOVEL STRUCTURED LIGHT METHOD FOR ONE-SHOT DENSE RECONSTRUCTION
}

\author{
Sergio Fernandez and Joaquim Salvi \\ Institute of Informatics and Applications, University of Girona, \\ Av. Lluis Santalo S/N, E-17071 Girona (Spain). [sergiofn@eia.udg.edu]
}

\begin{abstract}
Dense acquisition for moving scenarios represents an active field of research in Structured Light (SL). A common solution is to project a single one-shot fringe pattern, extracting depth from the phase deviation of the imaged pattern. This implies the use of a phase unwrapping algorithm, which can fail in the presence of depth discontinuities and occlusions. Our work presents a new one-shot dense pattern where DeBruijn and Windowed Fourier Transform are combined obtaining a dense, absolute, accurate and computationally fast $3 \mathrm{D}$ reconstruction regarding the other existing techniques.
\end{abstract}

Index Terms - structured light, fringe analysis, active stereo, dense reconstruction, computer vision.

\section{INTRODUCTION}

Three dimensional measurement constitutes an important topic in computer vision. A main classification is done according to whether the object surface is touched or not to perform the measurement (which respresents a constraint in many applications). Among non-contact measuring systems, active methods based on SL are composed of one or more digital cameras and a Digital Light Projector (DLP). DLP projects a designed pattern to impose the illusion of texture onto the measuring surface, increasing the number of correspondences, thus being able to perform measurements even in presence of textureless surfaces [1]. Among the different SL techniques, the ability to measure moving surfaces (up to the acquisition time of the camera) is only achieved by one-shot patterns. DeBruijn and M-arrays-based patterns perform one-shot absolute reconstruction with good accuracy at the expense of acquiring a sparse (feature wise) measurement ([5], [7], [5], [12], [6]). Besides, fringe-based patterns achieve one-shot dense (pixel wise) reconstruction, though innacuracies can occur at surface discontinuities due to the non-absolute (periodic) coding intrinsic to the method [9]. There exist some techniques that obtain density and absolute coding by using one-shot spatial grading [2] [10], but both achieve a rather low accuracy [9]. These problems are overcome by the proposed one-shot dense pattern, which combines the accuracy and absolute coding of DeBruijn techniques with the density of the Windowed Fourier Transform.

\section{MAIN FREQUENCY-BASED TECHNIQUES}

The idea of frequency based techniques is to extract the depth directly from the phase deviation of the imaged pattern. Fourier Transform, Windowed Fourier Transform and Wavelet Transform have been traditionally used for this purpose. In Fourier Transform (FT) a sinusoidal grating was projected onto the measuring surface, and the reflected deformed pattern was recorded [11]. Once reflected, the phase component is modified by the shape of the surface as shown in eq. (1):

$$
I(x, y)=a(x, y)+c(x, y) e^{2 \pi i f_{\phi} y^{p}}+c *(x, y) e^{-2 \pi i f_{\phi} y^{p}}
$$
where

$$
c(x, y)=\frac{1}{2} b(x, y) e^{i \phi(x, y)}
$$

where $c *(x, y)$ is the complex value of constant $c(x, y)$ and contains the wrapped phase component, which is extracted and unwrapped. From this, the depth is extracted regarding the baseline and the distance to a reference plane. However, leackage errors appear due to the limited length of the analysed data. Windowed Fourier Transform (WFT) was proposed to solve this problem. WFT splits the signal into segments before the analysis in frequency domain is performed. These segments must be small enough to reduce the errors introduced by boundaries, holes and background illumination, at the same time it must be big enough to hold some periods and hence allow the detection of the main frequency [3]. The 4-D coefficients $S f(u, v, \xi, \eta)$ provided by eq. (3) are used to estimate the frequency and the corresponding phase distribution using Windowed Fourier Filtering (WFF) or Windowed Fourier Ridge (WFR) [4].

$S f(u, v, \xi, \eta)=\int_{-\infty}^{\infty} \int_{-\infty}^{\infty} f(x, y) \cdot g(x-u, y-v) \cdot \exp (-j \xi x-j \eta y) \mathrm{d} x \mathrm{~d} y$

Finally, in Wavelet Transform (WT) the window size increases when the frequency to analyse decreases, and viceversa, improving the avoidance of leackage effects. Similarly to WFT, a 4D transform is obtained from WT, following the same procedure to extract the phase deviation. In computational applications a dyadic net is used to generate the set of wavelet functions(that is, the size is modified by the factor $2^{j}$ ). This can be a drawback for fringe pattern analysis, where the change in the spatial fringe frequencies throughout the image is not high enough to produce a relative variance of $2^{j}$ in the size of the optimal wavelet. 


\section{PROPOSED TECHNIQUE}

\subsection{Pattern creation}

The proposed pattern consists in a colored sinusoidal fringe pattern, where the color of the different fringes follows a DeBruijn sequence. A k-ary DeBruijn sequence of order $\mathrm{n}$ is a circular sequence $d_{0}, d_{1}, d_{n^{k}-1}$ (length $n^{k}$ ) containing each substring of length $k$ exactly once (window property of $k$ ). In our approach we set $n=3$ as we work only with red, green and blue colors. We set the pattern to have 64 fringes, regarding the pixel resolution of the projector and the camera, that is $n^{k}>=64$ therefore $k=4$. An algorithm performing the sequence generation provides us an arbitrary DeBruijn circular sequence $d_{0}, d_{1}, . ., d_{80}$. The pattern, of size $m \times n$, is generated in the HSV space. The V channel contains a sinusoisal signal for every column, with discrete frequency $f=64 / n$. The $\mathrm{H}$ channel maps a value of the previously computed DeBruijn sequence to every period of the $\mathrm{V}$ channel. The $\mathrm{S}$ (saturation) channel is set to 1 for all the pixels.

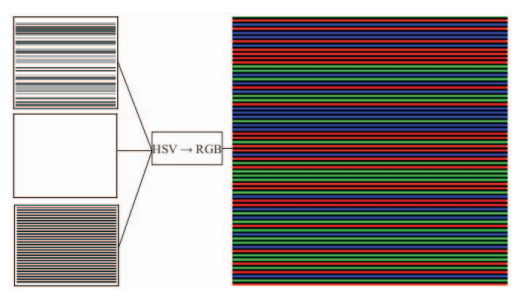

Fig. 1: Left: HSV channels for $m=64$. Right: RGB pattern.

\subsection{DeBruijn analysis}

First, color calibration is applied to the recovered image. Then, a maxima localization algorithm with sub-pixel accuracy looks for the center of the fringes in every color channel, in a similar way that is done for slit patterns ([1], [9]). The constraints of maxima-minima alternance of peaks and a total of $n=64$ periods are considered in the search. Also, peaks lower than the $70 \%$ of maximum peak are suppress to avoid false peaks detection. Finally, the color and position of the fringe maxima is obtained. Afterwards, a dynamic programming algorithm is applied to match projected and recovered color sequences [12].

\subsection{Windowed Fourier Transform analysis}

The WFT has been chosen for frequency fringes analysis, as it avoids leackage distortion and a more precise window width selection than in WT. An adapted Morlet wavelet is chosen for WFT analysis (eq. 4), as it provides good frequency and spatial localization at the same time [3].

$$
\Psi_{\text {Morlet }}(x)=\frac{1}{\left(f_{c}^{2} \pi\right)^{1 / 4}} \exp \left(2 \pi i f_{c} x\right) \cdot \exp \left(\frac{-x^{2}}{2 f_{b}^{2}}\right)
$$

where $f_{c}$ is the mother wavelet central frequency and $f_{b}$ is the window size frequency. Average period $(\mathrm{pm})$ and standard deviation $(s t d)$ are extracted from the single periods corresponding to each column on the $\mathrm{V}$ channel. The std represents the uncertainty in the estimated frequency, and is crucial to perform a global analysis of the image. The average frequency for the $m \times n$ pattern is computed as $f_{m}=n / p m$. The frequencies analysed are in the range $\left[f_{m}-2 \cdot s t d, f_{m}+2 \cdot s t d\right]$ in both $\mathrm{x}$ and $\mathrm{y}$ axes, where $f_{m}$ is the average frequency. Using this range the $95 \%$ of detected frequencies are analysed. The window size has been spammed from one up to three periods. Finally WFR is applied in this window range, obtaining the corresponding wrapped phase of the fringes.

\subsection{Merging DeBruijn and wrapped phase patterns}

Despite leackage avoidance intrinsic to WFT, still some smoothing is present in the wrapped phase. This is corrected shrinking or expanding it accordingly to the extracted DeBruijn slits with a $4^{t h}$ order non-linear interpolation. The corrected wrapped phase is then spammed in the DeBruijn correspondences map to create a full (dense) correspondences map. The 3D cloud of points is obtained from triangulation (projector-camera system must be previously calibrated). Finally, statistical and bilateral filtering are applied to the resulting data.
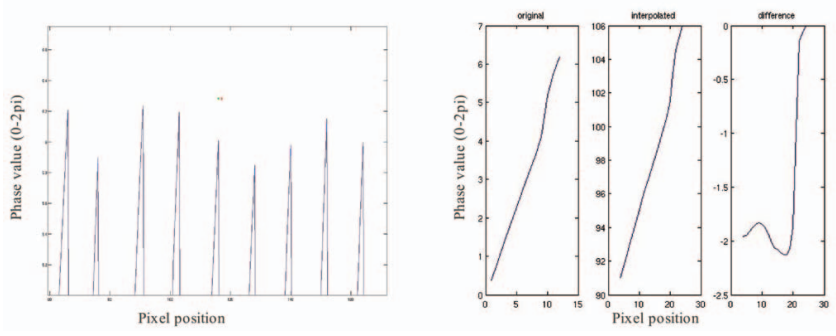

Fig. 2: Left: detail of the wrapped phase and a crest maxima (in red), and its corresponding slits line position (in green). Right: correction of the wrapped phase.

\section{IMPLEMENTATION AND RESULTS}

The proposed algorithm was implemented and compared with the techniques present in the work of Salvi et al. [9]. They correspond to the main groups existing in SL in both dense and sparse reconstruction. The setup used for the tests was composed of an DLP video projector (Epson EMP-400W) with a resolution of $1024 \times 768$ pixels, a camera (Sony 3CCD) and a frame grabber (Matrox Meteor-II) digitizing images at $768 \times 576$ pixels with $3 \times 8$ bits per pixel (RGB). The baseline between camera and projector was about $60 \mathrm{~cm}$. The algorithms were implemented in Matlab 7.3. The compared methods were re-programmed from the corresponding papers since codes were not available. 


\subsection{Simulation results}

The peaks function available in Matlab has become a benchmark in fringe pattern analysis [9]. Therefore, a noised version with gaussian zero mean and standard deviation of $0.05 \%, 0.1 \%, 0.15 \%$ and $0.2 \%$ was used as input and the reconstructed shape was compared with the original. A comparison with the other one-shot techniques selected in [9] was done. Fig. 3 shows the best performance of our algorithm,

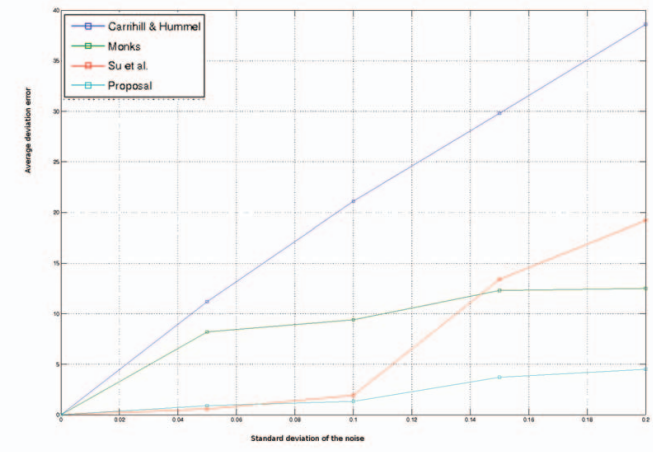

Fig. 3: Normalized depth error, for different values of noise.

whereas Carrihill and Hummel performs the worst. Besides, errors in the slits position of Monks approach are penalised by the low amount of reconstructed points. Among dense reconstruction techniques, Su et al. fails for $s t d>0.1$ due to the error in the unwrapping algorithm cause by noise slopes.

\subsection{Empirical results}

Quantitative results were analysed reconstructing a white plane at a distance of about $80 \mathrm{~cm}$ in front of the camera. PCA is used to span the $3 \mathrm{D}$ cloud of points onto a $2 \mathrm{D}$ plane defined by the two eigenvectors corresponding to the two largest eigenvalues, and thus compute the error. As can be extracted

\begin{tabular}{|c|c|c|c|c|}
\hline Technique & Average $(\mathrm{mm})$ & Stdev $(\mathrm{mm})$ & 3D Points & Patterns \\
\hline Monks et al. & 1.31 & 1.19 & 13899 & 1 \\
Posdamer et al. & 1.56 & 1.40 & 25387 & 14 \\
Guhring & 1.52 & 1.33 & 315273 & 24 \\
Pribanic et al. & 1.12 & 0.78 & 255572 & 18 \\
Carr.and Hummel & 11.9 & 5.02 & 202714 & 1 \\
Proposed technique & 1.18 & 1.44 & 357200 & 1 \\
\hline
\end{tabular}

Table 1: author's name of the technique; average error; standard deviation; reconstructed 3D points; projected patterns. As explained in [9], Su et al. is not conceived for this test.

from table 1, our proposal obtains one of the best accuracy results in terms of average and standard deviation of the error, similar to the algorithm of Monks et al. [5]. Among dense reconstruction techniques the method of Pribanic et al. [8] performs better, but requires a total of 18 projected patterns. Qualitative results were pursued reconstructing several 3D lambertian objects placed at $80 \mathrm{~cm}$ of the camera (see Fig. 4).

\section{CONCLUSION}

Most of the works presented in SL during the last years are based on frequency multiplexing approaches, as a way to obtain one-shot dense reconstruction. However, the periodicity in the recovered phase imposes a limit for depth reconstruction [4]. The proposed algorithm merges DeBruijn coding and frequency-based WFT to achieve dense reconstruction and absolute coding. The proposal was implemented and compared both quantitatively and qualitatively with some representative techniques of Structured Light. Simulated and empirical results showed the good performance of the proposed technique in terms of resistance to noise and accuracy of a reconstructed plane, similar to those obtained in DeBruijn sparse reconstructions.

\section{REFERENCES}

[1] J. Batlle, E. Mouaddib, and J. Salvi. Recent progress in coded structured light as a technique to solve the correspondence problem: a survey. Pattern Recognition, 31(7):963-982, 1998.

[2] B. Carrihill and R. Hummel. Experiments with the intensity ratio depth sensor. Computer Vision, Graphics, and Image Processing, 32(3):337-358, 1985.

[3] S. Fernandez, M. A. Gdeisat, J. Salvi, and D. Burton. Automatic window size selection in windowed fourier transform for $3 \mathrm{~d}$ reconstruction using adapted mother wavelets. Optics Communications, 284(12):2797-2807, 2011.

[4] Q. Kemao. 2D windowed Fourier transform for fringe pattern analysis: principles, applications and implementations. Optics and Lasers in Engineering, 45(2):304-317, 2007.

[5] T. Monks, J. Carter, and C. Shadle. Colour-encoded structured light for digitisation of real-time 3D data. In IEE 4th International Conference on Image Processing, pages 327-30, 1992.

[6] R. Morano, C. Ozturk, R. Conn, S. Dubin, S. Zietz, and J. Nissano. Structured light using pseudorandom codes. IEEE Transactions on Pattern Analysis and Machine Intelligence, 20(3):322-327, 1998.

[7] J. Pages, J. Salvi, C. Collewet, and J. Forest. Optimised De Bruijn patterns for one-shot shape acquisition. Image Vision and Computing, 23:707-720, 2005.

[8] T. Pribanic, H. Dapo, and J. Salvi. Efficient and Low-Cost 3D Structured Light System Based on a Modified NumberTheoretic Approach. EURASIP Journal on Advances in Signal Processing, Volume 2010, Article ID 474389, 11 pages, 2009.

[9] J. Salvi, S. Fernandez, T. Pribanic, and X. Llado. A state of the art in structured light patterns for surface profilometry. Pattern recognition, 43(8):2666-2680, 2010.

[10] J. Tajima and M. Iwakawa. 3-D data acquisition by rainbow range finder. In Pattern Recognition, 1990. Proceedings., 10th International Conference on, volume 1, pages 309-313, 1990.

[11] M. Takeda M, Mutoh. Fourier transform profilometry for the automatic measurement of 3-D object shapes. Appl. Opt, 22:3977-3982, 1983.

[12] L. Zhang, B. Curless, and S. Seitz. Rapid shape acquisition using color structured light and multi-pass dynamic programming. In 3D Data Processing Visualization and Transmission,, pages 24-36, 2002. 


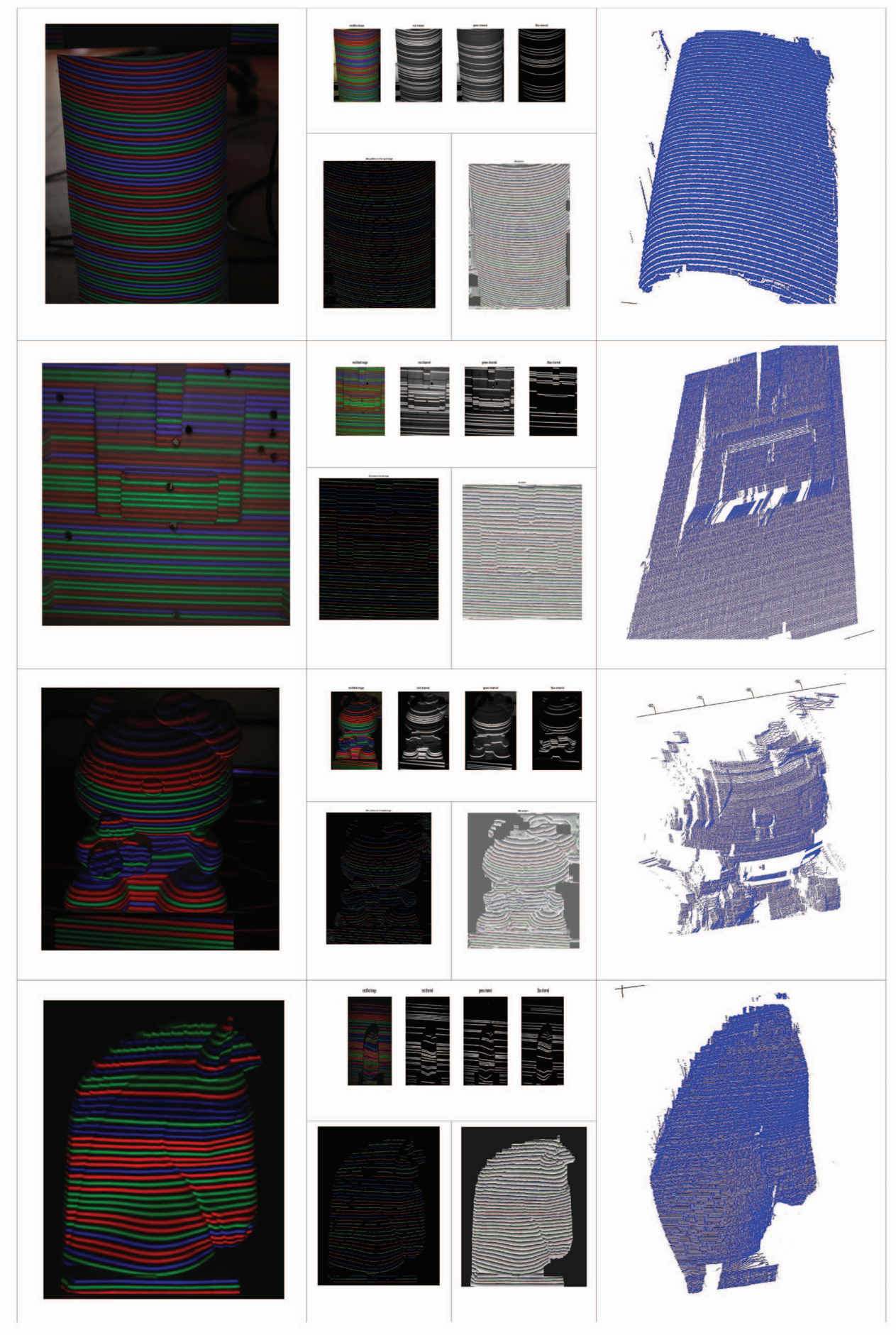

Fig. 4: For every object: on the left, input image. On the center the rectified extracted colors channels, and the slits and fringes extraction. On the right the recovered 3D cloud of points, where noised (occluded) regions have been filtered. 